\title{
Highlights from the literature
}

\section{PREGNANCY OUTCOMES AFTER CHILDHOOD KIDNEY TRANSPLANT}

As a trainee in the 1980s, I remember caring for a preterm infant who was the result of what was said to be the UK's first successful pregnancy in a renal transplant recipient. Things have moved on, and transplanted women can now confidently expect to become mothers. But can we be as optimistic about girls transplanted while still children? A report based on the Australia and New Zealand Dialysis and Transplant Registry says we can (Wyld ML et al. JAMA Pediatr 2015. doi:10.1001/ jamapediatrics.2014.3626) Using data on all renal transplants done in those countries between 1963 and 2012, the authors identified 101 pregnancies in 66 women transplanted as children ( $<18$ years), and 626 pregnancies in 401 women transplanted as adults. They compared these groups and found no significant differences between them for a range of important outcomes: in both groups live birth rates were around $76 \%$, and both groups had similarly high rates of pre-eclampsia $(28 \%)$, preterm birth (c. 50\%<37 weeks) and intra-uterine growth restriction. Similarly there was no difference in rates of deterioration of renal function after pregnancy. Gratifyingly, over the whole 5 -decade era, pregnancy termination rates for child-transplanted women dropped from $50 \%$ to zero, perhaps reflecting increased confidence in a successful outcome. Thus we can reassure the parents of a young girl facing a transplant that in spite of the recognised hazards of poor renal function, surgery, and many years of immunosuppression, they have a good chance of becoming grandparents.

\section{NEW GENETICS IN NEUROMUSCULAR DISORDERS}

There are many early-onset neuromuscular disorders with a heritable cause, but diagnosing them can be challenging: even with a muscle biopsy there can be doubt. It is important to get it right, to give accurate genetic counselling and because some conditions, such as congenital myasthenia, are amenable to drug treatment. Novel genetic techniques are well-suited to help with this, and using a 'gene screen' to investigate children with unexplained muscle weakness might prove useful. Researchers from South Korea recruited 43 children presenting with muscle weakness and/or hypotonia before age 5 years (Chae JH, et al. J Med Genet 2015;52:208-16). As well as traditional investigations (40/43 had a muscle biopsy) they undertook Next Generation Sequencing using a custommade probe to look for candidate mutations. This led to a firm diagnosis in an impressive 21/43, and possible candidate mutations in a further 7. Muscular dystrophies were the most common (14/43), followed by congenital myopathies and congenital myasthenia. As expected using this technology, they threw up some mutations whose pathological signficance was unknown (4/43). The authors argue that this technique is superior to other techniques such as whole exome sequencing, and that its use at an early stage may produce a precise diagnosis without the need to subject the child to a muscle biopsy.

\section{MORTALITY IN ADHD}

Children with attention deficit hyperactivity disorder (ADHD) are notoriously accidentprone, but does this translate into an increased long-term risk of death? It would require a huge study population to confirm this, and this is exactly what Danish researchers were able to do (Dalsgaard S et al. Lancet 2015;6736:61684-6). Denmark's national registry can link diagnostic data for the whole population with death records. Between 1981 and 2013 they identified over 32000 individuals of all ages with a formal diagnosis of ADHD and related behavioural disorders from a dataset containing 1.92 million people. Of those with ADHD, 179 died, which gave a mortality rate per 10000 person-years of $5 \cdot 85$, compared to 2.21 in those without the diagnosis (mortality rate ratio after adjusting for confounding factors 2.07: $95 \%$ CI $1 \cdot 70-2 \cdot 50 ; \mathrm{p}<0 \cdot 0001)$. Of 79 with ADHD for whom cause of death was known, $54(68 \%)$ were from unnatural causes, mostly accidents; overall the mortality risk for unnatural death was 3 times greater than for those without. The relative mortality increased with age, and most of the deaths were in those aged over 18 . Interestingly, although girls were fewer in number, their mortality risk was even higher than boys. Not surprisingly, co-morbidities of oppositional defiant disorder, conduct disorder and substance abuse disorder increased the risk, but even when these were excluded the overall risk remained significant. They had no data on stimulant treatment, but there was nothing to suggest that adverse effects due to medication were responsible for any deaths. Stimulant drugs were relatively little used in Denmark over this time period. The obvious next step is to look or any reduction in mortality in those on treatment.

\section{ENFORCED REST AFTER HEAD INJURY}

Some clinicans advise young people discharged from hospital after significant head injury to rest completely for a few days, in the belief that it will reduce symptoms of concussion. Is this necessary? A study from Wisconsin, USA, suggests not (Thomas DG et al. Pediatrics 2015. doi: 10.1542/peds.2014-0966). They randomised 88 young people aged 11 to 22 years who had presented to a paediatric emergency department within 24 hours of a head injury: cases were told to rest strictly for 5 days, while the controls received 'usual care', i.e. 1 to 2 days rest followed by gradual return to normal activity. All underwent neurocognitive and balance assessments at 3 and 10 days following the injury: there were no differences between the groups in these outcomes. However symptom diaries showed that the strict rest group actually reported more postconcussive symptoms, such as headache and dizziness (mean symptom score 188 vs. 132; $\mathrm{p}<0.03$ ), perhaps because they had nothing else to do but think about how they felt.

Many current head injury management guidelines (including those from the UK's National Institute for Health and Care Excellence, NICE CG176) do not specify a recommended rest period. Perhaps they should actively encourage a speedy return to normal activity.

\section{FINALLY...}

Lucina is always happy to receive suggestions for articles from peer-reviewed journals to feature in 'Highlights'. If you have any please contact me on info.adc@bmj. com 